\title{
Setup for the Measurement of Apparent Mass Matrix of Standing Subjects
}

\author{
Marco Tarabini, Stefano Solbiati, Bortolino Saggin, and Diego Scaccabarozzi
}

\begin{abstract}
This paper describes a system for the measurement of the apparent mass (AM) matrix of standing subjects. The system uses two electrodynamic shakers to generate vibrations along two mutually perpendicular axes (vertical and horizontal) and allows the identification of the full AM matrix with two tests, in which the standing subject is exposed to vertical excitation combined in turn with fore-and-aft and lateral vibration. A 3-D force platform measures the forces and the torques transmitted from the vibrating platform to the feet. The force platform, set up with piezoelectric load cells, has been designed in order to obtain a measurement bandwidth of $20 \mathrm{~Hz}$. The supports of different load cells are meant to minimize bending moments on the sensors and to minimize the axes crosstalk. The force platform has been calibrated with a least-squares approach, using reference masses and a dynamometric hammer. The AM uncertainty, evaluated through the experiments' repeatability and reproducibility, is lower than $\mathbf{3 . 4 \%}$ along the three axes (confidence level $68 \%)$. The measurement bandwidth is $20 \mathrm{~Hz}( \pm 2 \%)$ and the crosstalk between orthogonal axes is lower than $5 \%$, in accordance with the design requirements.
\end{abstract}

Index Terms-Biodynamic response, force platform, multiaxial vibration, vibration, WBV.

\section{INTRODUCTION}

$\mathbf{S}$ EVERAL studies showed evidence that vibration transmitted to the body generates cyclic stresses that may be critical to the spine [1]-[3]. Consequently, Rakheja et al. [4], [5], Matsumoto and Griffin [6], Huang and Griffin [7], and Tarabini et al. [8] studied the resonances of different body parts since a better understanding of the physiological mechanisms concurring with the biodynamic response may improve the mathematical models and the comprehension of spine degenerative phenomena. The biomechanical response of the human body is usually expressed in terms of apparent mass (AM), which requires the measurement of the force and the acceleration at the driving point (i.e., the point through which the vibration is transmitted to the body). The AM has relevant applications not only in the study of pathologies related to vibration exposure but also in the study of interactions between civil structures and the human body [9]. The forces transmitted at the driving point are generally measured by load cells supporting a rigid metal plate, whose inertial contribution is later subtracted from the measured forces.

Manuscript received December 22, 2015; revised February 14, 2016; accepted March 12, 2016. Date of publication April 20, 2016; date of current version July 12, 2016. The Associate Editor coordinating the review process was Dr. Domenico Grimaldi.

The authors are with the Department of Mechanical Engineering, Politecnico di Milano, Lecco 23900, Italy (e-mail: marco.tarabini@ polimi.it).
At the current state of the art, the biodynamic response of standing subjects has been mainly studied by observing the response (inertial force) along the same axis of the stimulus (acceleration) either in the vertical [4], [8], [10] or in the fore-and-aft direction [11]. The force on the horizontal plane (fore-and-aft and lateral direction) deriving from a vertical acceleration was studied in [12]; Subashi et al. [12] evidenced that the response along the transverse axis might be comparable to those aligned with the vibration stimulus. The experimental setup was based on an electrodynamic shaker (used to create vertical vibration) and a triaxial force platform (used to measure forces in the vertical and fore-and-aft directions); the force platform used four triaxial quartz piezoelectric load cells. There are several standards that focus on the biodynamic response of the human body [13]-[15], but none of them focuses on the response of standing subjects.

Our work is motivated by the fact that to date, there are no studies focused on the measurement of the AM matrix of standing subjects. Only four elements of the AM matrix have been identified (three diagonal terms plus the horizontal response to a vertical stimulus), and consequently, it is not possible to predict the response of the body under multiaxial vibration. In addition, the diagonal terms of the AM matrix have been measured on different groups of people. Moreover, all existing studies were carried out under single-axis excitation. One of the reasons for which the full AM matrix of the human body has never been studied is that the measurement setup allows the generation of vibration along horizontal and vertical axes and the simultaneous measurement of force along three mutually perpendicular axes. Since the cross-axis terms are expected to be smaller than the diagonal terms, the metrological characteristics of the 3-D force platform are critical: the design requirements for the measurement system include the following:

1) a passband of at least $20 \mathrm{~Hz}$ (typical for the study of biodynamic response of the human body);

2) a standard measurement uncertainty lower than $5 \%$ (to detect the cross-axis contributions that are expected to be negligible above $5 \mathrm{~Hz}$ );

3) a crosstalk between the measurement axes as low as possible.

In order to measure the nine components of the AM matrix, we have designed a setup that uses two single-axis shakers to generate random vibrations along two mutually perpendicular axes. Given that the excitation is provided by two shakers, the identification of the AM matrix requires two different tests for each subject (one aligned with the vibration generated by the 
horizontal shaker and the other lateral). Given the particular design requirements, we have also chosen to design a sixcomponent load cell; the configuration was selected among the ones existing in the literature [16]-[23], i.e., the cross-based force/torque sensors, the Stewart platform-based force/torque sensors, and the custom force/torque sensors. For instance, the configuration proposed in [16] used a setup based on a Maltese cross made of aluminum and 16 strain gauges measuring both forces and torques; two measurement systems were used to identify the forces and torques at the forefoot and the rear foot. The measurement of the total force at the $2 \mathrm{ft}$ required 64 strain gauges, thus entailing a complex measurement chain not suitable to our problem. Another possible approach is based on the Stewart platform [20]; Hou et al. [23] optimized the configuration of a prestressed six-component force sensor, analyzing the position and orientation errors. Dai and Kerr [17] described a six-component load cell that used strain gauges to measure the bending moments on the supports of the six platforms' legs. Although there are several works that have used the hexapod configuration, its main limitation is the large height of the setup, which limits its use in our case where the six-component load cell has to be mounted on the top of an electrodynamic shaker.

In other studies, scholars proposed six-component load cells with custom design: for instance, Joo et al. [18] described a configuration based on a double parallel plate system and a ring load cell. The structural elements were designed with a finite-element model in order to obtain similar strain sensitivities for all the six components; the cross-axis sensitivity was $3.8 \%$ of the full scale. In general, the beam configuration is not suitable in the presence of high bending moments, owing to the limited bending stiffness of this setup. Consequently, we have chosen to adopt a configuration similar to the one described in [22], [24], and [25], in which piezoelectric load cells are mounted on selectively compliant supports. The main advantage of the proposed method is the possibility of modifying the distance between the supports in order to increase the bending stiffness and to obtain a wide bandwidth in the presence of high moments of inertia characterizing the human body.

This paper is structured as follows. Section II describes the design of the triaxial force plate and of the dual-axis excitation system. Section III describes the metrological characterization of the setup. Preliminary experimental results are presented and discussed in Section IV. Conclusions of this paper are drawn in Section V.

\section{Method}

The AM is defined as the ratio between the force $(F)$ and the acceleration $(a)$ spectra. AM can be computed by measuring both the force and the acceleration at the point where the vibration is transmitted to the body. Since both $F$ and $a$ are vectors, the $\mathrm{AM}$ is a $3 \times 3$ matrix in which the diagonal terms express the inertial forces aligned with the direction of vibration and extra-diagonal terms indicate the inertial forces along directions perpendicular to the imposed vibration. The identification of $\mathrm{AM}$ therefore requires the measurement of the force and acceleration vectors. Given that

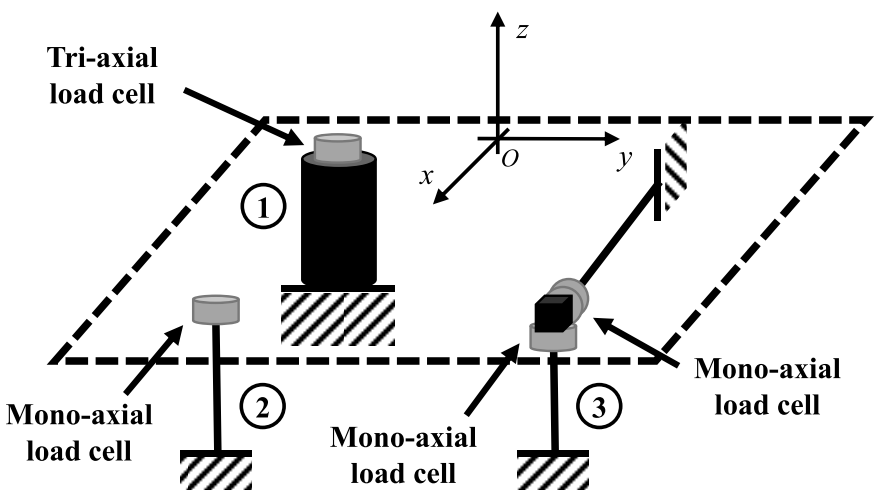

Fig. 1. Proposed measurement scheme.

the supporting plate is translating, the acceleration vector can be measured by a triaxial accelerometer; the force vector can be measured with a force platform, whose characteristics must comply with the previously evidenced design requirements.

\section{A. Force Plate Design}

The force plate has been designed to measure the forces transmitted by the feet of the subjects at the driving point along three orthogonal axes. The load cells' positions are constrained by the interface of the shaker available in our laboratory (LDS V830). We have chosen to adopt an isostatic mounting condition, which is the one that usually grants the lowest crosstalk between the axes. The supports' scheme has been designed with a 3-D hinge and two rollers (one monodimensional and the other 2-D) similarly to what has been done in other applications [22], [24]. Rollers have been obtained using thin mechanical elements, with stiffness along one direction much larger than those along other directions. Four piezoelectric load cells were used to measure the inertial forces according to the scheme shown in Fig. 1. The triaxial load cell (PCB 260A11) is located between the rigid support and the plate. Three monoaxial load cells (PCB 211B) are mounted on compliant supports in order to be exposed to reaction forces aligned with their measurement axes.

A 3-D model of the supports and plate assembly was created using the software PTC Creo Parametric. A finite-element model was used to verify the static resistance of the compliant elements and to ensure that the natural frequencies of the system accomplish with the design requirements. The load cells were modeled as 3-D springs with the stiffness matrix derived from the manufacturer datasheet.

Both the supports' lengths and cross-sectional areas were chosen imposing a stiffness ratio between the compliant supports and the rigid ones. The supports' stiffness was computed imposing external forces of $1 \mathrm{kN}$ along each axis and computing the reaction forces and the displacements at the load cell-support interface. The stiffness along the compliant directions was at most $1 \%$ of that of the rigid elements in the same direction.

The supporting plate $\left(500 \times 500 \mathrm{~mm}^{2}\right.$ in size, $5 \mathrm{~mm}$ thick with stiffening ribs) was designed to maximize the 

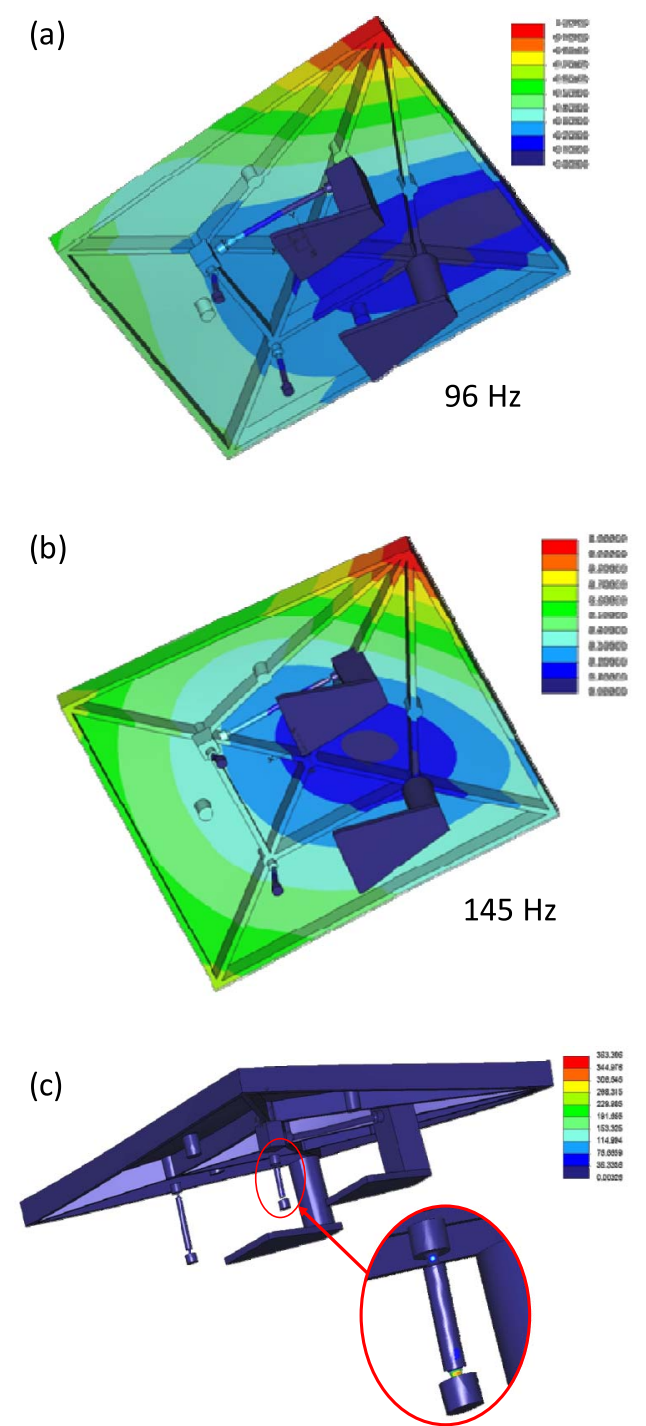

Fig. 2. Results of FE analyses. (a) and (b) Modes of vibration of the plate. (c) Maximum Von Mises stress.

measurement bandwidth; the geometry of the ribs was constrained by the shaker interface and was optimized in order to have the maximum resonance frequency, retaining the overall mass of $15 \mathrm{~kg}$. The modal analysis outlined that the first two vibration modes of the unloaded force plate have frequencies of 96 and $145 \mathrm{~Hz}$ [Fig. 2(a) and (b)]. The presence of standing subjects could be modeled by introducing a frequency-dependent mass on the feet contact area; however, this procedure is cumbersome and the AM of standing subjects is lower than the static mass above $10 \mathrm{~Hz}$. Consequently, tests were performed with a lumped mass of $80 \mathrm{~kg}$ on the feet contact area, obtaining an underestimation of the actual system bandwidth. In these conditions, the first two natural frequencies were, respectively, 56 and $102 \mathrm{~Hz}$, i.e., more than twice the desired bandwidth of the measurement system $(20 \mathrm{~Hz})$.

The supports' static resistance was verified by considering both the inertial force under maximum acceleration and the subject weight. The maximum force occurs at approximately $5 \mathrm{~Hz}$, where the $\mathrm{AM}$ of standing subjects

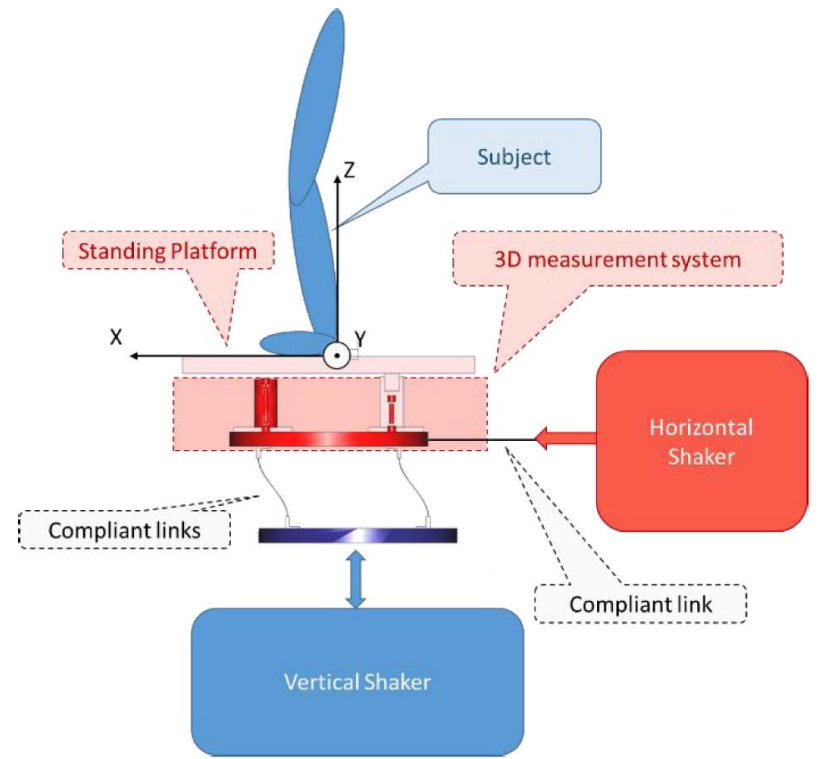

Fig. 3. Scheme of the dual-axis excitation system.

is nearly three times the static mass of the subject [8]. Considering a maximum acceleration of $3.5 \mathrm{~m} / \mathrm{s}^{2} \mathrm{rms}$ $\left(5 \mathrm{~m} / \mathrm{s}^{2} 0\right.$-peak) and a maximum subject static mass of $110 \mathrm{~kg}$, the maximum load (static+dynamic) is approximately $2800 \mathrm{~N}$. Given the rigid behavior of the assembly in the low-frequency region, a static analysis was run with an equivalent force of $2800 \mathrm{~N}$ instead of the combination of static and dynamic loads as in [26]. The largest Von Mises stress (385 MPa) occurred at the undercuts of the compliant supports (margin of safety versus yield 1.2), as shown in Fig. 2(c).

\section{B. Dual-Axis Interface}

As previously outlined, the identification of the AM matrix requires excitation along the horizontal axis direction and thus requires the simultaneous use of (at least) two shakers, one with a horizontal axis and the other with a vertical axis [27]. In this way, the AM matrix can be measured with two tests by asking the subject to rotate $90^{\circ}$ on the platform.

The force plate described in the previous section has been mounted onto an interface designed to be compliant along the secondary direction of excitation. The interface consists of two disks of aluminum joined together by two thin plates of spring steel (EN 10270-1 class SM), as shown in Fig. 3. Similar to the case of the 3-D force platform, the design requirements were resistance under mechanical loads during the tests and the passband frequency larger than $20 \mathrm{~Hz}$.

A 3-D model was parametrized versus the distance between the upper and lower disks, the spring steel plates' size, distance, and thickness. Values that allowed achieving the design requirements are presented in Table I.

The equivalent Von Mises stresses were computed by imposing on the upper disk the maximum displacement at the highest acceleration magnitude along the horizontal and vertical directions. As in the previous case, the standing subject was modeled with a vertical force of $2800 \mathrm{~N}$; since the AM along the horizontal axis is much smaller than the 
TABLE I

COMPLiAnt ELEMENTS

Parameter

Distance between the upper and the lower discs

Width of the spring steel plates

Distance between the two spring steel plates

Thickness of the spring steel plates

one along the vertical axis [28] and given the limited force provided by the horizontal shaker, the horizontal forces were not included in the analysis. The stresses on the compliant elements of the dual-axis interface were not critical, given that the displacement along the compliant direction was driven by the lateral shaker, and the elements were sized to achieve low stiffness to avoid force loss. The compression stress deriving from the vertical load besides, is minor being the blades were sized by the buckling condition. The first vibration mode with the unloaded dual-axis interface connected to the two shakers was $92 \mathrm{~Hz}$, thus satisfying the design requirements.

\section{Final Setup}

The force platform and the dual-axis interface are shown in Fig. 4: the force platform (with the load cells) was fixed to the dual-axis interface and then mounted on the vertical shaker head. The load cells' signals were conditioned using Bruel \& Kjaer Nexus conditioning amplifiers (with bandpass filters from 0.1 to $100 \mathrm{~Hz}$ ). The signals of IEPE accelerometers were conditioned by Bruel \& Kjaer Nexus amplifiers with the same bandpass filters, in order to avoid phase distortions evidenced in similar applications [29]. Signals were sampled using National Instruments hardware (NI 9234 modules in a cDAQ chassis); the sampling frequency was $2048 \mathrm{~Hz}$; signals were stored on a personal computer and analyzed offline.

The vibration of the vertical shaker (LDS V830, maximum stroke $\pm 25 \mathrm{~mm}$ ) was controlled in closed loop by an LMS Test.Lab system. The vibration of the horizontal shaker (Tira VIB, maximum stroke $\pm 12 \mathrm{~mm}$ ) was controlled in closed loop by a Data Physics hardware. The accelerometers (Bruel \& Kjaer 4508 B and PCB 356A21 with a nominal sensitivity of $10 \mathrm{mV} / \mathrm{ms}^{-2}$ ) were individually calibrated using a Bruel \& Kjaer 4294 calibrator (reference acceleration $10.02 \mathrm{~m} / \mathrm{s}^{2} \mathrm{rms}$ at $159.15 \mathrm{~Hz}$, standard uncertainty lower than $1 \%)$.

\section{Calibration Procedure}

Piezoelectric transducers are sensitive to bending at the mounting surface and have nonnull cross-axis sensitivities. In the presence of dual-axis excitation and with nonideal constraint conditions of each sensor, the adoption of the manufacturer-declared sensitivities might lead to a large uncertainty in the identification of the 3-D force vector. The 3-D force platform underwent a metrological characterization procedure consisting of identification of the sensitivity
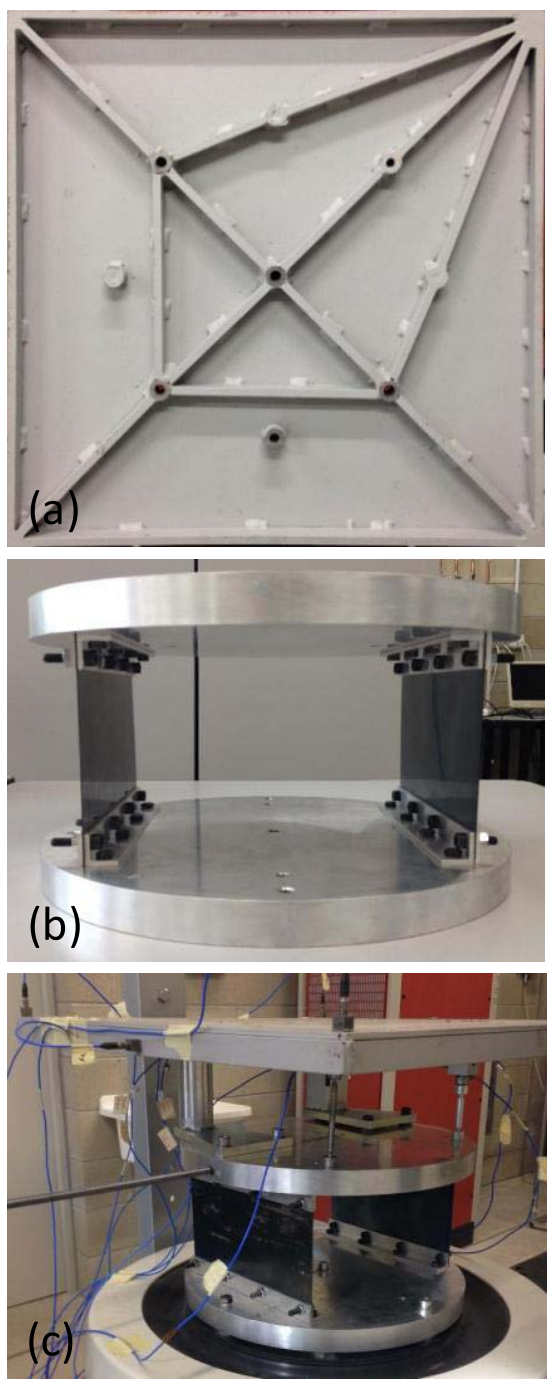

Fig. 4. Pictures of (a) standing platform, (b) compliant support, and (c) final experimental setup.

matrix A derived by solving the calibration problem

$$
F=\mathbf{A} \cdot F_{\mathrm{NOM}}
$$

where $F$ contains the actual loads applied during calibration, $F_{\text {NOM }}$ refers to the loads measured by the load cells with their nominal sensitivities, and the sensitivity matrix $\mathbf{A}$ includes the correction coefficients accounting for the mounting conditions and the system geometric characteristics. Instead of determining the exact solution with the minimum number of tests, we opted to find a solution minimizing the difference A $\cdot F_{\mathrm{NOM}}-F[30]$ for a redundant set of conditions.

Generally, $\mathbf{A}$ is a full matrix: the elements on the main diagonal are the sensitivities along the desired directions, while other elements are the transverse-axis sensitivities. Dealing with force platforms, the input quantities should be either forces or torques, both evaluated in a proper Cartesian reference system. Since during the measurement process the reference parameter is the inverse of the sensitivity matrix $\mathbf{S}$, 
the calibration problem was formulated as follows:

$$
\left[\begin{array}{l}
F_{x} \\
F_{y} \\
F_{z} \\
M_{x} \\
M_{y} \\
M_{z}
\end{array}\right]=[S]_{6 \times N} \cdot\left[\begin{array}{llll}
F_{\mathrm{NOM}, 1} & F_{\mathrm{NOM}, 2} & \ldots & F_{\mathrm{NOM}, N}
\end{array}\right]
$$

where $N$ is the number of channels to calibrate, which in our case is 6 , and $F_{\mathrm{NOM}, 1} \ldots F_{\mathrm{NOM}, N}$ are the forces measured by load cells with their nominal sensitivity. The elements of the $\mathbf{S}$ matrix indicate the possible differences between the ideal conditions and the real ones.

$F$ elements have been generated in two ways.

1) In the first series of tests, forces and torques were the inertial contributions of calibrated masses fixed on the vibrating platform; with this method (hereinafter referred to as stationary), it is possible to calibrate the measurement axes aligned with the two shakers' axes.

2) Given that the platform can vibrate in the directions aligned with the two shakers, the third direction was calibrated with forces and torques generated by an impact hammer hitting the standing platform in different positions; these tests are called nonstationary.

Stationary tests were performed by placing masses from 5 to $40 \mathrm{~kg}$ on the plate in different positions (in order to generate different moments). The vibration was a random Gaussian noise with rms accelerations of 0.5 and $1.0 \mathrm{~ms}^{-2}$ between 1 and $20 \mathrm{~Hz}$. The net AM was derived by subtracting the complex FRF measured in idle conditions from the sum of the three load cells' readings [8], [31]. When large masses were positioned far from the plate center, the influence of transverse (lateral) resonance of the shaker mobile mass was important in the bandwidth of interest. The sensitivity was therefore derived in the range of $2-15 \mathrm{~Hz}$, where the FRF was constant in magnitude $( \pm 7 \%)$; this limitation does not affect the experiments, where subjects always stood in the central position (and the am of the subject is always lower than $20 \mathrm{~kg}$ above $10 \mathrm{~Hz}$ ).

The nonstationary tests were performed by hitting the edge of the force plate along horizontal directions with an impact hammer (PCB 086C02). The location of the hammering points was aligned with two load cells in order to limit the bending moments; the impact direction was aligned with the $x$ and $y$ measurement axes. The deviation from the nominal sensitivity has been evaluated with the modulus of the $H_{1}$ estimator of the frequency response function in the frequency range between 2 and $20 \mathrm{~Hz}$. The phase was always lower than $5^{\circ}$ and therefore considered null to ease the computations.

Tests were also performed to assess the experiments' repeatability (evaluated by repeating measurements with calibrated masses placed in different positions) and reproducibility (analyzed by changing the orientation of load cells to evaluate the impact of transversal sensitivity and mounting imperfections on measurement uncertainty).

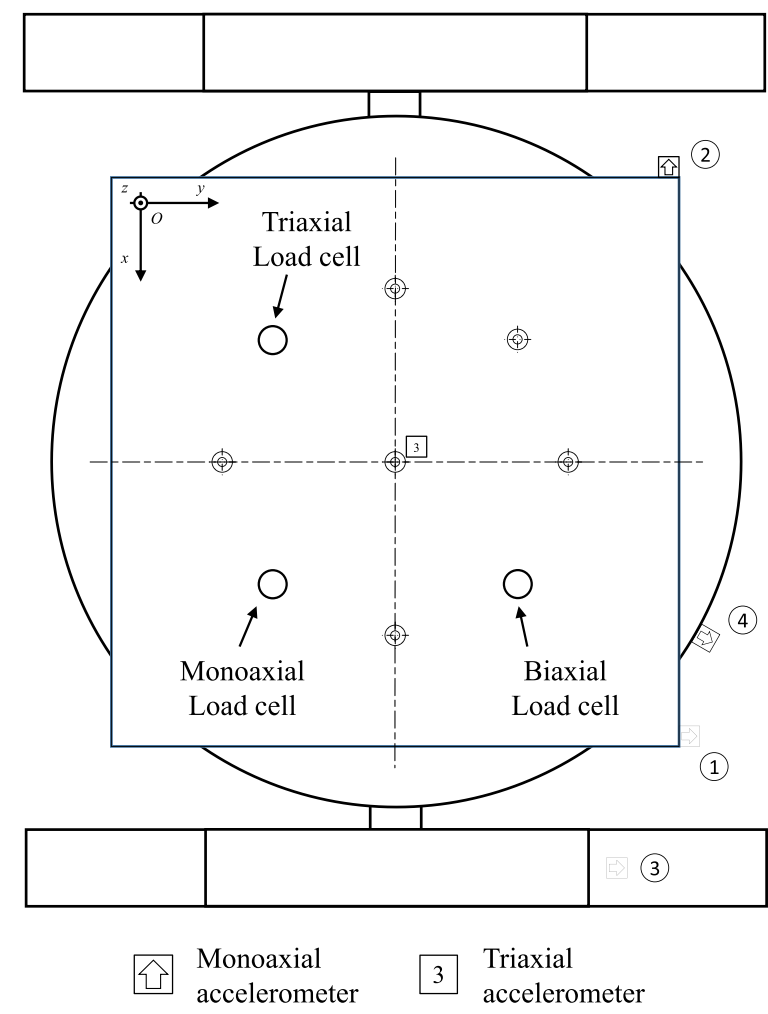

Fig. 5. Experimental setup for the identification of the frequency response function.

\section{RESULTS}

\section{A. Bandwidth}

The validity of the results obtained by the modal analysis of the FE model was checked by mounting the force plate onto an electrodynamic shaker to verify that the resonances were outside the frequency range of interest. The force plate was exposed to random vertical vibration (magnitude $1.5 \mathrm{~ms}^{-2} \mathrm{rms}$ between 1 and $150 \mathrm{~Hz}$ ). The experimental setup is shown in Fig. 5: five accelerometers (four monoaxial and one triaxial) were fixed on the force plate, the chassis, and the supporting frame of the shaker.

The bandwidth was verified by analyzing the frequency responses between the vertical acceleration (stimulus) and the accelerations measured by the triaxial accelerometer placed at the plate center (responses). The results (Fig. 6) showed two resonances at 102.8 and $126.6 \mathrm{~Hz}$ (compatible with those evidenced by the FE analysis) and minor resonances at 50.5, 61, and $72 \mathrm{~Hz}$. These resonances were related to the rotation of the plate along the vertical axis and therefore not critical to the AM measurement. The following are the values of the FRF modulus inside the frequency band of interest $(1-20 \mathrm{~Hz})$ :

1) between 0.98 and 1.01 (vertical axis);

2) lower than 0.05 ( $x$-axis);

3) lower than 0.02 ( $y$-axis).

\section{B. Calibration of the Triaxial Force Plate}

The calibration was performed using the inertial forces generated both by calibrated masses under known accelerations 


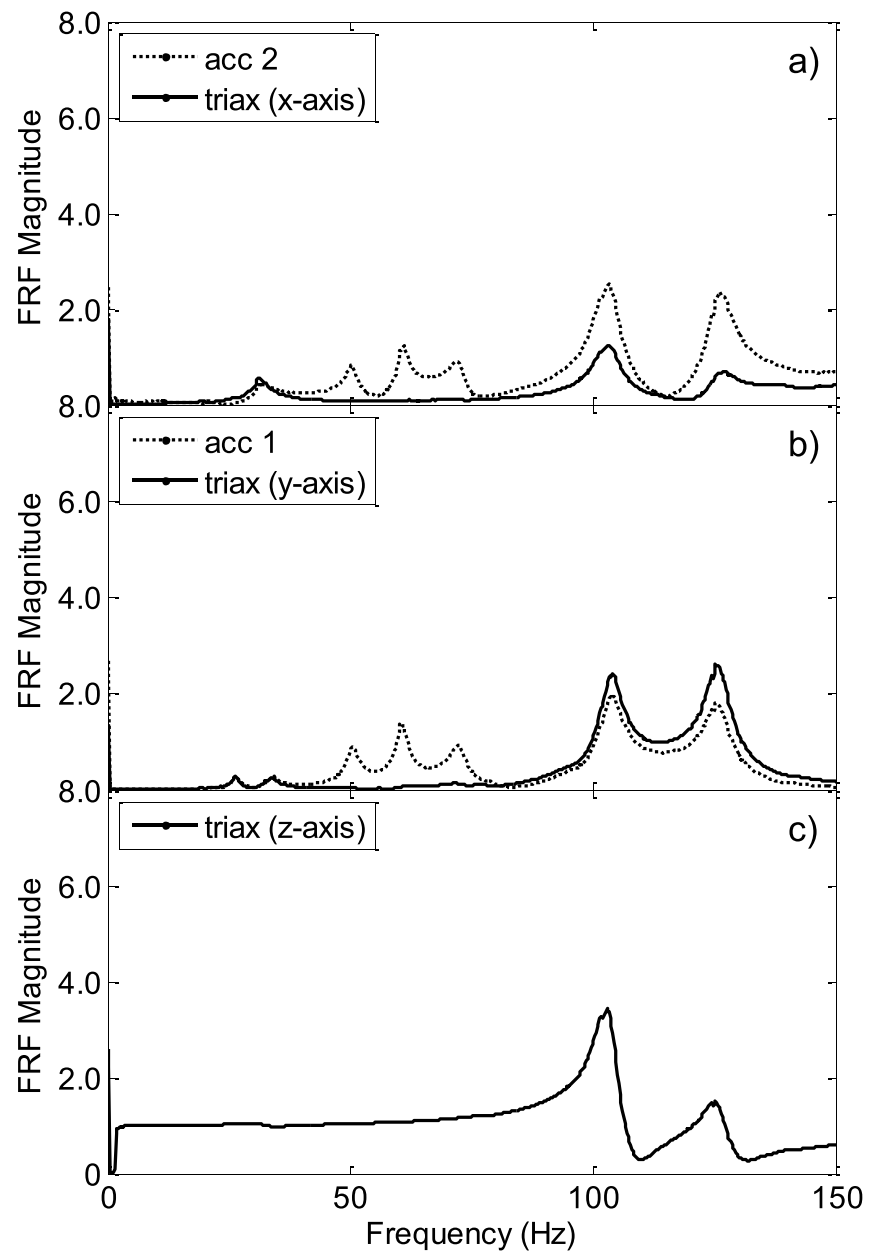

Fig. 6. Vibration transmissibility between the vertical stimulus and the response along the (a) $x$-axis, (b) $y$-axis, and (c) $z$-axis.

and by the impact hammer. The resulting sensitivity matrix accounts for both the nonideal behavior of the transducers and the crosstalk between the measurement axes

$\left[\begin{array}{cccccc}S_{F x, z 1} & S_{F x, x 2} & S_{F x, z 2} & S_{F x, x 3} & S_{F x, y 3} & S_{F x, z 3} \\ S_{F y, z 1} & S_{F y, x 2} & S_{F y, z 2} & S_{F y, x 3} & S_{F y, y 3} & S_{F y, z 3} \\ S_{F z, z 1} & S_{F z, x 2} & S_{F z, z 2} & S_{F z, x 3} & S_{F z, y 3} & S_{F z, z 3} \\ S_{M x, z 1} & S_{M x, x 2} & S_{M x, z 2} & S_{M x, x 3} & S_{M x, y 3} & S_{M x, z 3} \\ S_{M y, z 1} & S_{M y, x 2} & S_{M y, z 2} & S_{M y, x 3} & S_{M y, y 3} & S_{M y, z 3} \\ S_{M z, z 1} & S_{M z, x 2} & S_{M z, z 2} & S_{M z, x 3} & S_{M z, y 3} & S_{M z, z 3}\end{array}\right]$
$=\left[\begin{array}{cccccc}0.05 & 1.13 & 0 & 1.15 & 0.17 & 0.02 \\ 0.09 & 0.15 & 0 & 0.23 & 1.22 & 0.04 \\ 0.98 & 0 & 1.03 & 0 & 0 & 1.41 \\ 3.80 & 0 & 225.76 & 0 & 0 & 18.54 \\ 244.21 & 0 & 219.55 & 0 & 0 & 7.37 \\ 22.42 & 293.38 & 2.81 & 25.82 & 22.72 & 7.60\end{array}\right]$.

The matrix can be split into two $3 \times 6$ submatrices, the first one allowing the computation of the three forces $F_{x}, F_{y}$, and $F_{z}$ and the second (not interesting for the identification of AM) allowing the computation of the three torques $M_{x}$, $M_{y}$, and $M_{z}$. The terms lower than $1 \%$ were numerically set to zero; numerical values of the torque submatrix are larger given that they include the moment arms expressed in millimeters.

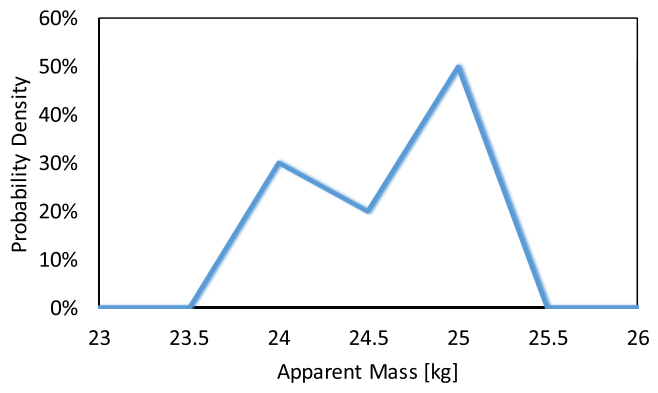

Fig. 7. Distribution of AM measured with the 25-kg deadweight.

The uncertainty in the measurement of vertical loads has been evaluated by fixing calibrated masses in the range from 5 to $40 \mathrm{~kg}$ on the plate; the vertical acceleration was a random stimulus with an rms value of $1 \mathrm{~m} / \mathrm{s}^{2}$ (white noise). Experiments with each mass were repeated ten times, moving the masses in different positions. The uncertainty in acceleration was derived from that of the accelerometer calibrator and was $0.7 \%$ of the measurement. The uncertainty in mass (declared by the manufacturer) was $0.1 \%$. The AM uncertainty was computed as the rms value of the difference between the AM estimated with (2) and (3) and the actual mass. The measured AM was obtained by dividing the force by the measured acceleration. The worst case was represented by a mass of $25 \mathrm{~kg}$, where there was a systematic underestimation (average measured mass $24.3 \mathrm{~kg}$ ) and a standard deviation of measurement of $0.4 \mathrm{~kg}$. The rms of the difference between the actual mass and the one estimated by the measurement system was $0.8 \mathrm{~kg}$, i.e., $3.2 \%$ of the nominal mass. The measurement uncertainty ( $3.4 \%$ in the worst case of $25 \mathrm{~kg}$ ) was obtained by combining the rms relative uncertainty $(3.2 \%)$ with the reference mass uncertainty and the acceleration uncertainty. The distribution of the measured AM is shown in Fig. 7.

The uncertainty in the $x y$ plane was derived as the deviation from measurements performed with the dynamometric hammer (uncertainty declared by the manufacturer was lower than $1 \%$ ) and was $2.3 \%$ along the $x$-axis and $2 \%$ along the $y$-axis.

The cross-axis sensitivity has been evaluated in the tests performed on the shaker by computing the lateral force deriving from a vertical acceleration. Results differed depending on the mass location: the errors on the lateral forces ( $x$ and $y$ ) were $1 \%$ and $3 \%$ of the vertical force when the mass was centered; errors increased to $4 \%$ and $7 \%$ when the mass was located close to the borders; values are similar to those reported in the existing studies focused on six-component force platforms [18]. As already outlined for the bandwidth analysis, the limitation is not relevant for AM analyses, given that during the tests subjects are required to stand with the center of gravity reasonably close to the plate center.

The effect of noise of the measurement chain was evaluated by acquiring the forces and accelerations measured in idle conditions; the maximum rms value of force along the measurement axes (in the frequency band 2-20 Hz) was $0.1 \mathrm{~N}$. The rms of acceleration in idle conditions was $6 \mathrm{~mm} / \mathrm{s}^{2}$. 


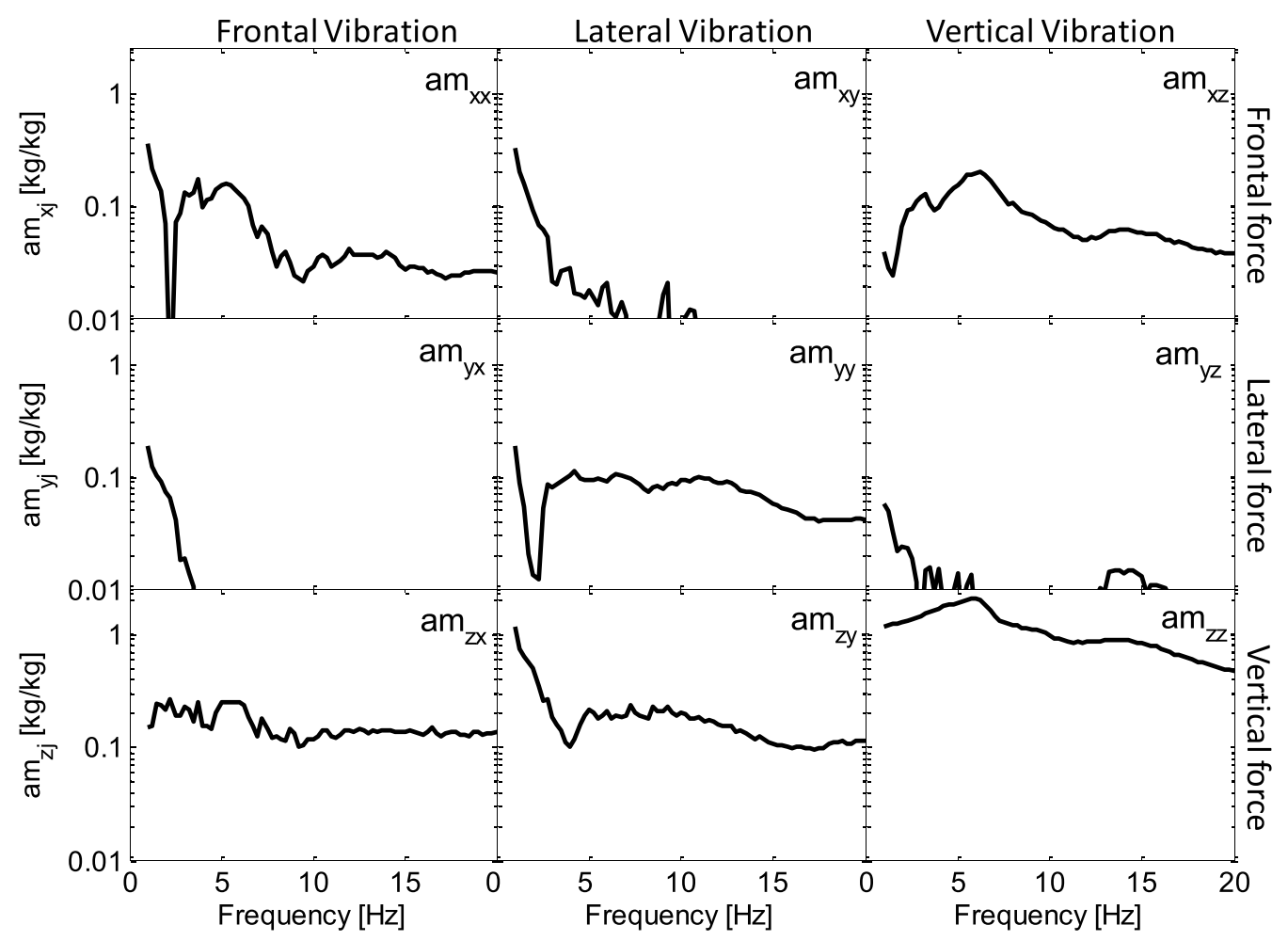

Fig. 8. Example of AM matrix measured from preliminary tests. The first letter indicates the direction of force and the second indicates the direction of acceleration.

The effect of measurement noise was trivial with respect to linearity errors evidenced in the previous calibration phases.

\section{Preliminary Tests}

Preliminary tests were performed in order to verify the validity of the proposed approach for the identification of the AM matrix, which is alternatively identified by imposing the vibration $\left(0.2 \mathrm{~m} / \mathrm{s}^{2} \mathrm{rms}\right)$ along the $x, y$, and $z$ axes. During the experiments, the subject stood on the force plate by adopting an upright position with straight legs, keeping his feet $25 \mathrm{~cm}$ apart. The subject wore his own shoes and was advised to look straight at a fixed point during the trial and to maintain the same position without moving the body.

The results, expressed in terms of normalized AM, i.e., the ratio between AM and the subject weight, are shown in Fig. 8. The plots show that the most important contribution is the vertical force deriving from a vertical acceleration $\left(\mathrm{am}_{z z}\right)$. The other two diagonal terms $\left(\mathrm{am}_{y y}\right.$ and $\left.\mathrm{am}_{x x}\right)$ are lower than 0.1 at frequencies higher than $5 \mathrm{~Hz}$ and are comparable to the cross-axis terms $\mathrm{am}_{z y}$ and $\mathrm{am}_{z x}$. Lateral forces $(Y$-direction) deriving from vertical $(Z)$ and frontal $(X)$ stimuli are insignificant (am lower than 0.02 at frequencies above $5 \mathrm{~Hz}$ ). Similarly, the frontal force deriving from lateral vibration is less than 0.01 above $5 \mathrm{~Hz}$.

The results, although limited to a single subject, are consistent with the ones reported in [4], [11], and [12]; the results were obtained by testing one axis at a time. A statistical analysis performed on different subjects is presented in [28].

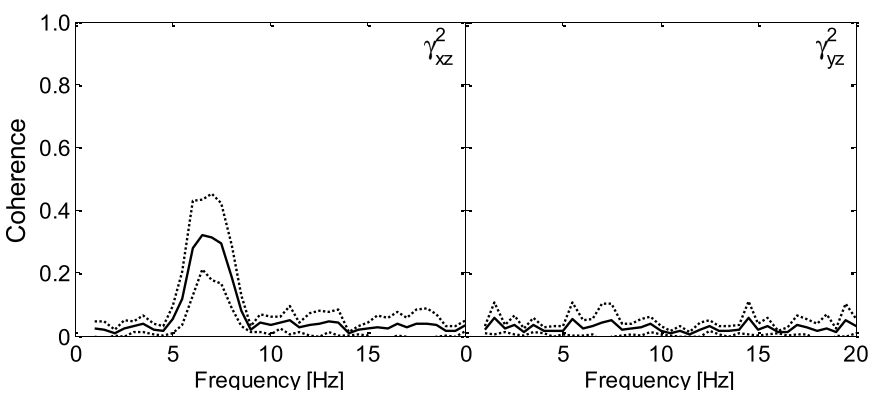

Fig. 9. Coherence functions between the accelerations along perpendicular axes.

The coherence between vibrations along two mutually perpendicular axes is shown in Fig. 9; the use of two independent controllers provides for uncorrelated vibrations along the perpendicular directions. The coherence between the vertical and lateral directions is always lower than 0.1 ; the coherence between the vertical and frontal directions is higher than 0.1 only in the range between 5 and $10 \mathrm{~Hz}$, with a maximum at a frequency close to $7 \mathrm{~Hz}$. In this range, there is a strong coupling effect exerted by the human body (and not by the measurement system, as outlined in the calibration).

\section{DISCUSSION}

Experimental results showed that the proposed experimental setup meets the design requirements for the measurement of the human body $\mathrm{AM}$ in a standing position in 
the presence of single-axis or dual-axis excitations upon controlling the vibration along two mutually perpendicular axes.

The main difficulty in the design of the system was the design of a force platform with a bandwidth of $20 \mathrm{~Hz}$ and a limited cross-axis sensitivity. The design requirements were achieved by mounting the load cells on selectively compliant mechanical elements. The 3-D force platform was mounted on a dual-axis interface, designed to create independent vibrations along two perpendicular axes using the two shakers available in our laboratory.

The main limitation of the proposed setup is that the identification of AM requires two tests, since it is possible to create vibrations only along two axes at a time. The limitation could be overcome by adding a third shaker and substituting the steel blades of the dual-axis interface with flexible beams to allow a planar motion of the upper plate. The use of three shakers would also enable comparison of the AM matrices identified under simultaneous triaxial vibration and under one-axis-at-a-time vibration. This could be interesting, since preliminary studies showed that when combining vertical and horizontal accelerations, AM components along the two directions are different from those derived under single-axis vibrations.

The adopted calibration procedure leveraged both calibrated masses and a force hammer for the identification of the sensitivity matrix. The achieved uncertainty of the proposed measurement system is lower than $3.4 \%$ along the vertical axis and $2 \%$ along the horizontal axis. These values are small in comparison with the typical intersubject and intrasubject variabilities, which are approximately $10 \%$ and $20 \%$ [8]. The measurement bandwidth (greater than $20 \mathrm{~Hz}$ according to the modal analysis) is sufficient to describe AM, where all the terms except $\mathrm{am}_{z z}$ are lower than 5\%. If it is necessary to know $\operatorname{am}_{z z}$ above $20 \mathrm{~Hz}$, it is possible to measure it with a conventional single-axis measurement system like the one used in previous experiments [3], [8].

The results of the tests performed on a single subject displayed that the AM cross-axis terms are comparable to the diagonal terms: $\mathrm{am}_{x z}$ and $\mathrm{am}_{x z}$ are, in some frequency ranges, even larger than $\operatorname{am}_{x x}$ and $\mathrm{am}_{y y}$, and they cannot be neglected. These results only describe the biomechanical response of a single subject, but the results of statistical analyses performed on a wider population [28] confirmed the preliminary findings presented here.

The next step of the research will be the analysis of torques measured by the six-component platform to derive useful information about the importance of the fore-and-aft motion of the body when exposed to linear vibration.

\section{CONCLUSION}

In this paper, we have described a setup for the measurement of the AM matrix of standing subjects. The setup uses two electrodynamic shakers to generate 2-D vibration and requires two tests to identify the full AM matrix. The setup was designed to minimize the axes crosstalk, given that the cross-axis AM terms are generally lower than the diagonal terms. Particular care was taken during the calibration of the
3-D force platform, which was performed using the leastsquares approach. The calibration evidenced that the design requirements were fulfilled; the setup has a measurement bandwidth of $20 \mathrm{~Hz}$ and a measurement uncertainty lower than $3.4 \%$ when the subject is standing in the central position of the force plate. Preliminary tests allowed the measurement of AM of a single subject. The results showed that the crossterms including the vertical stimulus or response are larger than the diagonal terms in the fore-and-aft or lateral direction. This suggests that the mere use of diagonal terms does not provide for a comprehensive description of the dynamic behavior of the human body, with implications in modeling the effect of people on civil structures.

\section{REFERENCES}

[1] M. Bovenzi, M. Schust, G. Menzel, J. Hofmann, and B. Hinz, "A cohort study of sciatic pain and measures of internal spinal load in professional drivers," Ergonomics, vol. 58, no. 7, pp. 1088-1102, 2014.

[2] M. Bovenzi, "A longitudinal study of low back pain and daily vibration exposure in professional drivers," Ind. Health, vol. 48, no. 5, pp. 584-595, 2010.

[3] M. Tarabini, B. Saggin, and D. Scaccabarozzi, "Whole-body vibration exposure in sport: Four relevant cases," Ergonomics, vol. 58, no. 7, pp. 1143-1150, 2015.

[4] S. Rakheja, R. G. Dong, S. Patra, P.-É. Boileau, P. Marcotte, and C. Warren, "Biodynamics of the human body under whole-body vibration: Synthesis of the reported data," Int. J. Ind. Ergonom., vol. 40 pp. 710-732, Nov. 2010.

[5] S. Rakheja, J. Z. Wu, R. G. Dong, A. W. Schopper, and P.-É. Boileau, "A comparison of biodynamic models of the human hand-arm system for applications to hand-held power tools," J. Sound Vib., vol. 249, pp. 55-82, Jan. 2002.

[6] Y. Matsumoto and M. J. Griffin, "Comparison of biodynamic responses in standing and seated human bodies," J. Sound Vib., vol. 238, pp. 691-704, Dec. 2000.

[7] Y. Huang and M. J. Griffin, "Nonlinear dual-axis biodynamic response of the semi-supine human body during vertical whole-body vibration," J. Sound Vib., vol. 312, nos. 1-2, pp. 296-315, 2008.

[8] M. Tarabini, B. Saggin, D. Scaccabarozzi, D. Gaviraghi, and G. Moschioni, "Apparent mass distribution at the feet of standing subjects exposed to whole-body vibration," Ergonomics, vol. 56, no. 5, pp. 842-855, 2013.

[9] G. Busca, A. Cappellini, S. Manzoni, M. Tarabini, and M. Vanali, "Quantification of changes in modal parameters due to the presence of passive people on a slender structure," J. Sound Vib., vol. 333, no. 21, pp. 5641-5652, 2014.

[10] Y. Matsumoto and M. J. Griffin, "Mathematical models for the apparent masses of standing subjects exposed to vertical whole-body vibration," J. Sound Vib., vol. 260, pp. 431-451, Feb. 2003.

[11] Y. Matsumoto and M. J. Griffin, "The horizontal apparent mass of the standing human body," J. Sound Vib., vol. 330, pp. 3284-3297, Jun. 2011.

[12] G. H. M. J. Subashi, Y. Matsumoto, and M. J. Griffin, "Apparent mass and cross-axis apparent mass of standing subjects during exposure to vertical whole-body vibration," J. Sound Vib., vol. 293, pp. 78-95, May 2006.

[13] Mechanical Vibration and Shock-Free, Mechanical Impedance of the Human Hand-Arm System at the Driving Point, document ISO 10068, International Organization for Standardization, 1998.

[14] Mechanical Vibration and Shock-Range of Idealized Values to Characterize Seated-Body Biodynamic Response Under Vertical Vibration, document ISO 5982, International Organization for Standardization, 2001.

[15] Mechanical Vibration and Shock-Mechanical Transmissibility of the Human Body in the $z$ Direction, document ISO 7962, International Organization for Standardization, 1987.

[16] L.-P. Chao and C.-Y. Yin, "The six-component force sensor for measuring the loading of the feet in locomotion," Mater. Design, vol. 20, pp. 237-244, Oct. 1999.

[17] J. S. Dai and D. R. Kerr, "A six-component contact force measurement device based on the Stewart platform," Proc. Inst. Mech. Eng. C, J. Mech. Eng. Sci., vol. 214, no. 5, pp. 687-697, 2000. 
[18] J. W. Joo, K. S. Na, and D. I. Kang, "Design and evaluation of a sixcomponent load cell," Measurement, vol. 32, pp. 125-133, Sep. 2002.

[19] S. A. Liu and H. L. Tzo, "A novel six-component force sensor of good measurement isotropy and sensitivities," Sens. Actuators A, Phys., vol. 100, pp. 223-230, Sep. 2002.

[20] J. Zhenlin, G. Feng, and Z. Xiaohui, "Design and analysis of a novel isotropic six-component force/torque sensor," Sens. Actuators A, Phys., vol. 109, pp. 17-20, Dec. 2003.

[21] Y.-J. Li, J. Zhang, Z.-Y. Jia, M. Qian, and H. Li, "Research on force-sensing element's spatial arrangement of piezoelectric sixcomponent force/torque sensor," Mech. Syst. Signal Process., vol. 23 , pp. 2687-2698, Nov. 2009.

[22] B. Saggin, D. Scaccabarozzi, I. Shatalina, and M. Tarabini, "Experimental characterization of vibration sources," in Proc. IEEE Int. Instrum. Meas. Technol. Conf. (I2MTC), May 2012, pp. 2300-2305.

[23] Y. Hou, J. Yao, L. Lu, and Y. Zhao, "Performance analysis and comprehensive index optimization of a new configuration of Stewart six-component force sensor," Mech. Mach. Theory, vol. 44, pp. 359-368, Feb. 2009.

[24] G. Moschioni, B. Saggin, M. Tarabini, and E. Alberti, "Prediction of hand arm vibration exposure by tool characterization: A case study," in Proc. 11th Int. Conf. Hand-Arm Vibrat., Bologna, Italy, 2007, pp. 525-532.

[25] G. Moschioni, B. Saggin, and M. Tarabini, "Prediction of data variability in hand-arm vibration measurements," Measurement, vol. 44 pp. 1679-1690, Nov. 2011

[26] B. Saggin, M. Tarabini, and D. Scaccabarozzi, "Infrared optical element mounting techniques for wide temperature ranges," Appl. Opt., vol. 49, no. 3, pp. 542-548, 2010.

[27] G. Legnani, D. Tosi, I. Fassi, H. Giberti, and S. Cinquemani, "The "point of isotropy' and other properties of serial and parallel manipulators," Mech. Mach. Theory, vol. 45, no. 10, pp. 1407-1423, 2010.

[28] M. Tarabini, S. Solbiati, B. Saggin, and D. Scaccabarozzi, "Apparent mass matrix of standing subjects exposed to multi-axial wholebody vibration," Ergonomics, pp. 1-26, Nov. 2015. [Online]. Available: http://www.tandfonline.com/doi/full/10.1080/00140139.2015.1108459

[29] M. Tarabini, B. Saggin, D. Scaccabarozzi, and G. Moschioni, "The potential of micro-electro-mechanical accelerometers in human vibration measurements," J. Sound Vib., vol. 331, pp. 487-499, Jan. 2012.

[30] M. Planitz, "Inconsistent systems of linear equations," Math. Gazette, vol. 63, pp. 181-185, Oct. 1979.

[31] M. Tarabini, S. Solbiati, G. Moschioni, B. Saggin, and D. Scaccabarozzi, "Analysis of non-linear response of the human body to vertical wholebody vibration," Ergonomics, vol. 57, no. 11, pp. 1711-1723, 2014. 